\title{
CONSIDERAÇÕES E DESCONSIDERAÇÕES ACERCA DO GÊNERO (?) DE FICÇÃO CIENTÍFICA
}

\author{
Sônia Melo de Jesus Ruiz ${ }^{1}$
}

RESUMO: O presente artigo objetiva problematizar a conceituação de ficção científica enquanto gênero literário. A contextualização histórica das obras de ficção científica é seguida da apresentação, exemplificação e análise de elementos que James e Mendlesohn (2003) e Gary Westfahl (1999) concebem como "imediatamente reconhecíveis" nas obras sci-fi, debatendo, assim, as considerações e desconsiderações da ficção científica como gênero literário. $O$ texto discute também o fato da fiç̧ão ser questionada como gênero por ser parte da cultura popular de massa e não erudita, focando no grande alcance da ficção científica e valorizando a sua capacidade de poder transitar entre múltiplos espaços e mídias, mantendo seu caráter volátil, mutante e híbrido.

PALAVRAS-CHAVE: Gênero ficção científica. Cultura de massa. Revistas pulp.

Edward James e Farah Mendlesohn (2003), editores do The Cambridge companion to science fiction, apresentam uma cronologia na qual Utopia, escrito por Thomas More em 1516, marca o início dos livros de ficção científica, contrariando a consideração tradicional de que Frankenstein, escrito em 1818 pela inglesa Mary Shelley, é responsável por esse marco (Cf. ALDISS, 1976, p. 13-21; DE LA ROCQUE e TEIXEIRA, 2001, p. 13).

Assim como não há uma concordância² sobre qual obra estabelece 0 início das publicações, o mesmo acontece com a definição do que realmente é a ficção científica, se um gênero literário ou não. Este é o primeiro questionamento apresentado por James e Mendlesohn (2003, p. 1), escritores britânicos de ficção científica, que argumentam: "Ficção científica é menos um gênero - um corpus literário do qual uma pessoa pode esperar certos elementos de enredo e clichês específicos - do que uma discussão em andamento." Os acadêmicos britânicos afirmam que, se fosse um gênero, saberíamos o que esperar de um livro do estilo assim que o pegássemos, o que não ocorre, pois a ficção científica extrai suas estruturas de enredo de qualquer gênero disponível e, assim, cada livro pode ser identificado potencialmente com um desses gêneros (Cf. JAMES e MENDLESOHN, 2003, p. 3). Dessa forma, eles apresentam sua definição de

\footnotetext{
${ }^{1}$ Professora adjunta do departamento de Línguas e Literatura (Inglês) da UERJ. Possui graduação em Letras - Português/Inglês pela Universidade Federal Fluminense (2000), História pela UNIVERSO (2009), Mestrado em American Studies pela University of Massachusetts, Boston (2002 - bolsista da Fulbright) e doutorado em Letras (UFF, 2009). Email:smjruiz@yahoo.com.

${ }^{2}$ Outros escritores, como Patrick Brantlinger (1980, p. 32), também questionam ser Frankenstein a primeira obra de ficção científica, afirmando que outras pesquisas, como as de Robert Philmus (1970) indicam ser a obra de Francis Godwin, The man in the moon, de 1638, digna desse marco.
} 
fiç̧ão científica como sendo "um modo de escrita que parece existir na variação dos padrões e demandas de estabelecimentos literários e o mercado de massa" (JAMES e MENDLESOHN, 2003, p. 1). Com essa afirmação, James e Mendlesohn (2003) a posicionam como um fenômeno da produção literária, bem como de consumo.

No entanto, Lúcia de La Rocque e Luiz Antonio Teixeira (2001, p. 11) entendem a questão de maneira diferente e afirmam que a fiç̧ão científica é um "gênero literário que se volta para o mundo da ciência, incluindo aí sua organização e produção, ideais de conhecimento e avanços técnicos etc." Também concordam com eles vários outros autores, como Darko Suvin (1972), Stanislaw Lem et. al (1973), Joanna Russ (1975), Roger Luckhurst (1991), Greg Grewell (2001), Istvan Csicsery-Ronay Jr. (2002), entre outros.

Mesmo classificando a ficção científica como um modo de escrita, e não propriamente um gênero literário, James e Mendlesohn (2003) destacam alguns elementos que concebem como "imediatamente reconhecíveis" nas obras: um "sentido de assombro/espanto" [sense of wonder], "estranhamento cognitivo" [cognitive estrangement] ${ }^{3}$, o cenário como personagem, "presentismo" [presentism] e especulação, marginalidade das relações e alienação.

O sentido de assombro ou espanto é descrito como "uma apreciação do sublime, seja natural, como os anéis de Saturno, seja tecnológico: uma estação espacial ou nave espacial" (JAMES e MENDLESOHN, 2003, p. 3). Esse sentido manifestava-se nos títulos das primeiras obras e revistas de ficção científica, que eram repletos de adjetivos como "novo", "primeiro", "espantoso", "impressionante" (Cf. CSICSERY-RONAY JR., 2002; JAMES e MENDLESOHN, 2003) e descreviam ambientes que não soam familiares, como os encontrados abaixo:4:

Coalescence was only about half a billion standards ago. Just yesterday, cosmically speaking. It is the youngest planetary system we've had a chance to study. Trouble is that Alpha Draconulus isn't too stable, and tends to throw tantrums involving things like charged particles thick enough to walk on. Station Three has four Type XXV Takamistsu Phase Generators. I have seen all four go red during solar storms. During one storm, the chief engineer's pelt turned white overnight. [...] Why not put the station on the planet's surface? Fine, find the surface. The planet's

\footnotetext{
${ }^{3}$ Termo cunhado por Darko Suvin (1972).

${ }^{4}$ Os trechos da antologia Carmen Miranda's ghost is haunting space station three organizada por Don Sakers (1990) serão usados como exemplos das elementos "imediatamente reconhecíveis" citados por James e Mendlesohn (2003).
} 
crust hasn't quite jelled. Tectonically the planet rates 12.4 on the openended Sullivan scale (CLOUGH apud SAKERS, 1990, p. 8).

They stood in the vast central volume of Space Station Three, the central lobby, where crystal pillars spiraled up to weave a web of balconies and catwalks as fragile and unreal as ice sculptures, and onlookers as evidently unreal leaned against the polished railings and pretended not to stare (SHWARTZ apud SAKERS, 1990, p. 104).

A narrativa de ficção científica não seria científica sem as inúmeras referências às máquinas extraordinárias, aos astros, alienígenas e aos espetaculares avanços tecnológicos, como os descritos acima. Há um estranhamento por parte do leitor, apresentado "[...] a um mundo fictício [que] é dissonante da [sua] experiência de mundo" (JAMES e MENDLESHON, 2003, p. 5). É nesse tipo de fiç̧ão que, como afirmam James e Mendlesohn (2003, p. 5), "a metáfora se torna literal": frases como "você é de outro mundo!" ou "ele vive no mundo da lua" podem ser entendidas no seu sentido denotativo. As expressões insólitas e os neologismos ganham significado na fértil e criativa imaginação dos leitores, que muitas vezes preenchem a lacuna dessa dissonância com seus conhecimentos prévios, adquiridos com o tempo, por meio de leituras de outros textos de ficção científica e pela intertextualidade comum nesse modo de escrita.

O "presentismo" das obras de ficção científica se apresenta juntamente com o sentido de assombro e se refere à falta de qualquer sentido de consequência - "E o que acontecerá depois?". Por exemplo, não se deixa explicação do que virá após uma explosão, como ocorre no conto "Rolling down the floor", de Amanda Allen (apud SAKERS, 1990, p.170):

\footnotetext{
Thirty seconds later, before the first lifeboats were even boarded, Space Station Three blew. The explosion rocked not only the dimensions that the generators drew energy from. Like rending of ancient, rotted fabric, dimensional barriers sundered.

Floating amid the debris, Carmen Miranda rested her hand on Stephson's bobbing corpse, gave a contented smirk, and beckoned at the newly-opened rifts in space... and at what lay beyond.

Soon, there were a lot of new ghosts following Carmen Miranda into space - and on to the next station.
}

São vários os questionamentos sem respostas: o fantasma de Carmen Miranda descansará em paz? Haverá sobreviventes? Será que o fantasma alcançará outra estação espacial e também a destruirá? Como será a reação dos governos terrestres ao saberem que todo o dinheiro, o tempo e a tecnologia empregados na construção da estação viraram poeira? 
O "presentismo" descrito acima, portanto, relaciona-se diretamente com a especulação recorrente nas narrativas de ficção científica, porque o mistério ronda essas narrativas e produz divagações por parte do leitor, que se pergunta: "E se...?". Nesse sentido, a ficção científica se torna uma ficção especulativa, em que somente algumas alternativas são exploradas, e muitas outras são deixadas para questionamento posterior do leitor.

Outro aspecto importante da ficção científica é o fato de seu discurso ser focado nas relações entre o ser humano e o universo. Como descrevem James e Mendlesohn (2003, p. 9), "enquanto a ficção da mainstream escreve sobre a complexidade de relacionamentos inter-humanos, o discurso da ficção científica é sobre nosso relacionamento com o mundo e com o universo." É por isso que relacionamentos amorosos e sexuais são temas geralmente secundários nesse contexto, e a alienação é mais recorrente. Importam mais à fiç̧ão científica as guerras interplanetárias, as descobertas científicas e tecnológicas, os contatos alienígenas, entre outros temas, do que um tórrido romance entre personagens o que é muitas vezes impossível, dada a presença de diferentes espécies de seres alienígenas que possuem formas, valores e culturas incompatíveis com os humanos. Além do mais, a grandiosidade de outros conflitos que dizem respeito a toda a humanidade, civilização e/ou ao universo e podem afetá-los é muito mais relevante do que qualquer affair estelar.

Mas, se existem padrões "imediatamente reconhecíveis" nas obras de ficção científica, poderíamos, então, considerá-los como fazendo parte de um gênero literário, uma vez que gênero literário pode ser definido por "tendência para reunir, em uma classificação, as obras literárias onde a realidade aparece de um determinado modo, através de mecanismos de estruturação semelhantes" (SOARES, 1997. p. 7). Como esclarecem Joseph Childers e Gary Hentzi em The Columbia dictionary of modern literary and cultural Criticism, "as classificações de gênero (genre) [...] podem ser feitas de acordo com outras categorias, como tema ou cenário" (CHILDERS e HENTZI, 1995, p. 126). Dessa forma, a ficção científica pode ser classificada como um gênero literário pelo simples fato de haver um conjunto de obras que possuem características semelhantes, como as descritas anteriormente. $\mathrm{O}$ que se percebe, na verdade, é que toda a discussão sobre o 
fato de a ficção científica ser, ou não, um gênero literário é parte de um questionamento maior: o do valor literário da ficção científica.

Já em 1975, no periódico Science fiction studies, Joanna Russ inicia seu artigo "Towards an aesthetic of science fiction", com a seguinte pergunta: "A ficção científica é literatura?" A resposta é dada logo em seguida e é bem objetiva: "Sim" (RUSS, 1975, p. 112). Russ responde a uma pergunta que mexe com os críticos de ficção científica da época, pois o gênero, como argumenta Raymond Williams (1988, p. 357), tem sua origem nas "[...] revistas de ficção, que são raramente de muito interesse literário". Sendo assim, faz-se necessário investigar as origens consideradas não literárias da ficção científica.

Gary Westfahl (1999) apresenta um histórico da tradição popular da ficção científica entre os anos de 1926 e 1980, descrevendo as chamadas American pulp magazines ${ }^{5}$, pioneiras na publicação desse tipo de ficção. Em 1926, surgiu a primeira revista de ficção científica, Amazing stories, lançada por Hugo Gernsback (Fig.1). Esse autor, que proclamou e teorizou o gênero - a princípio chamado de "scientifiction" - destacou três elementos básicos: "um romance cativante" [charming romance], um "fato científico" e uma "visão profética", que tinham por finalidade proverem entretenimento, educação científica e inspiração (Cf. WESTFAHL, 1999, p. 189).

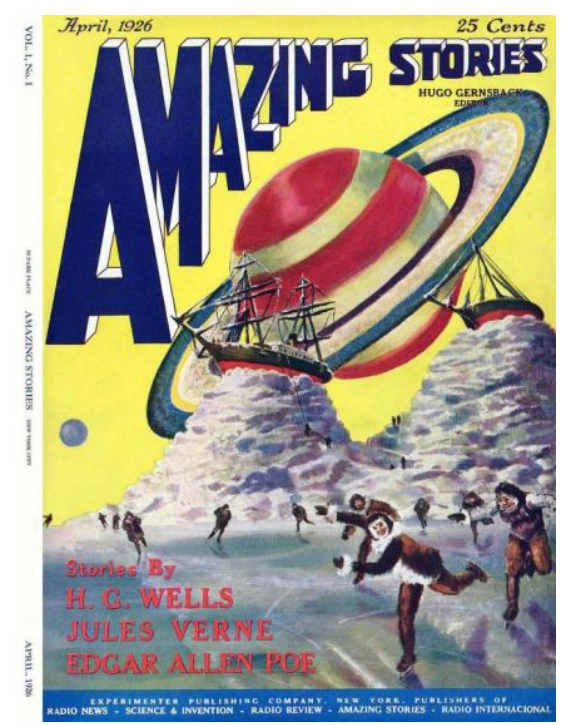

Fig. 1 Capa da primeira revista de ficção científica, Amazing Stories (Abril, 1926)

\footnotetext{
${ }^{5}$ De acordo com a Encyclopedia of Science Fiction, o nome "pulp" das revistas, se refere ao papel usado na publicação das mesmas. Bem ácido, áspero e com cheiro peculiar, o papel barato era feito de polpa de celulose tratada. (Cf. SFE, 2015)
} 
Em 1929, a revista Amazing stories ganhou um novo editor, T. O'Connor Sloane, menos preocupado com o aspecto didático da ficção científica - ao contrário de seu antecessor, que acreditava ser papel dessa forma de ficção estimular cientistas e inventores com novas ideias e especulações. Nessa mesma época, surgiram outras revistas do mesmo tipo, como a Wonder stories - fig. 2 que mais tarde seria a Thrilling wonder stories, e a Astounding stories superscience - fig. 3 - que depois se tornou apenas Astounding stories; tais publicações mostravam concordância com a visão de Sloane, já que davam mais relevância aos elementos de ação e de aventura do que aos científicos (Cf. WESTFAHL, 1999, p. 191).

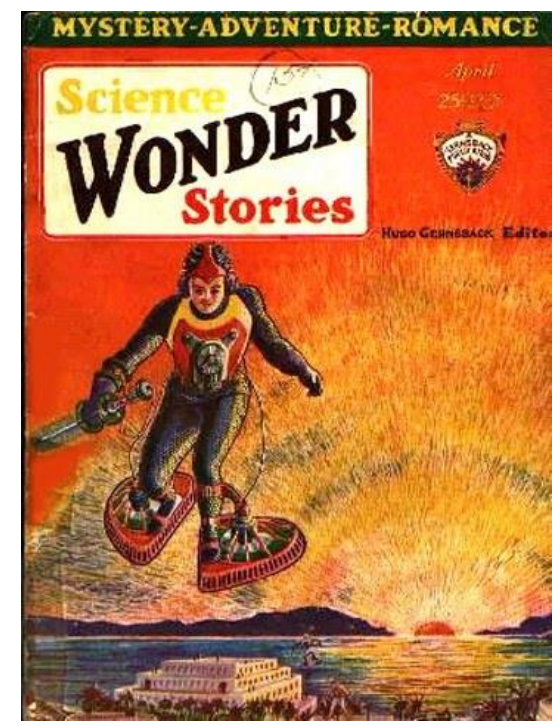

Fig. 2: Science Wonders Stories (Abril, 1930)

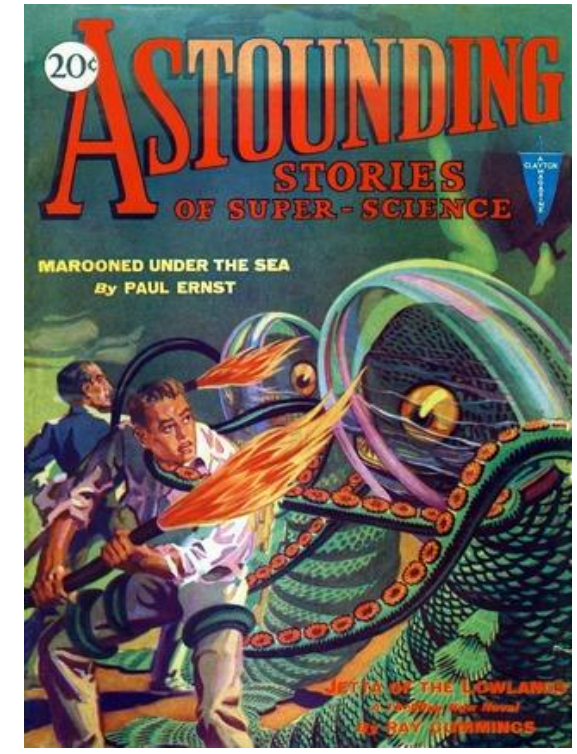

Fig. 3: Astouding Stories of Super Science (Setembro,1930) 
Em 1930, há um resgate dos ideais de Gernsback, por parte do editor $F$. Orlin Tremaine, da Astounding stories. Também nessa época, surgiram os fãclubes do gênero e fanzines, de qualidade editorial duvidosa.

John W. Campbell Jr. assumiu o controle editorial na Astounding stories em 1937 e manteve os princípios de "fiç̧ão, ciência e profecia", ampliando o conceito de ciência e incluindo áreas como as ciências sociais e a parapsicologia (Cf. WESTFAHL, 1999, p. 193). A explosão do gênero só se deu na década de 1950, quando houve a proliferação do material escrito e publicado de ficção científica, bem como a produção de filmes, o que deu visibilidade ao gênero e garantiu o aparecimento de novos autores, editores, antologistas e críticos.

Foi nessa época que surgiram as consagradas revistas The magazine of fantasy and science fiction e galaxy. Segundo Judith Merril, uma antologista de fiç̧ão científica proeminente em sua época, foi o editor da primeira, Anthony Boucher, que elevou a ficção científica a um novo status literário: "ele introduziu um conceito revolucionário no seu trabalho como editor: a ideia de que a ciênciafantasia [science-fantasy] [...] poderia ser bem escrita" (WESTFAHL, 1999, p. 195). O editor H. L. Gold, da Galaxy, também dava grande importância ao valor literário dos textos a serem publicados, afirmando que assim estava influenciando bons escritores de outros ramos literários a se aventurarem no gênero ficção científica (Cf. WESTFAHL, 1999, p. 196).

$\mathrm{O}$ fato de editores buscarem textos mais maduros literariamente, com uma competência técnica mais aprimorada, demonstra que o gênero gozava de um status questionável na crítica literária mainstream: ele era considerado "lowbrow". ${ }^{6}$ Sendo assim, constrói-se historicamente como um gênero literário de massa, direcionado a um público consumidor que, há mais de trinta anos, Stanislaw Lem (1977, p. 128) qualificou como popular:

They can count on some 200,000 steady readers, scattered across the
globe from New Zealand to Europe and Canada. These sci-fi fans-known
as "fandom" are not only great book buyers; they also publish specialty
magazines known as "fanzines" which are published in limited editions of
fifty to 500 copies. The pages of these periodicals warrant the attention of
sociologists, for they carry a high proportion of letters to the editor which
suggest that fandom is largely made up of frustrated individuals

${ }^{6}$ O termo, oriundo da prática pseudocientífica do século XIX de identificar tipos raciais e inteligências de acordo com o tamanho e formato craniano, significa alguém ou algo intelectual ou esteticamente inferior, oposto da expressão "highbrow" (Cf. LEVINE, 1988, p. 221-222). 
estranged from society. [...] Sci-fi readers are neither snobs, experts, intellectuals, bookworms, nor sophisticates. They are part of the mass culture market, a portion of which is captured by sci-fi along with beer and championship games.

É interessante destacar a comparação do consumo de ficção científica com o de jogos esportivos. A mesma analogia foi feita por Lawrence W. Levine para tratar da popularidade de Shakespeare nos EUA. Ele mostra que tanto as peças shakespearianas nos teatros do século XVIII e XIX quanto os atuais eventos esportivos eram e são populares por serem vistos por um público enorme e heterogêneo e também por serem de fácil acesso, numerosos e baratos, o que tornava e torna possível seu consumo por classes sociais menos favorecidas (Cf. LEVINE, 1988, p. 26). O mesmo aconteceu com as histórias de ficção científica: as revistas eram baratas por fazerem parte de edições simples, publicadas sem grandes revisões, em tamanho pequeno e em papel barato, com capa mole, e poderiam assim ser compradas por qualquer pessoa, o que não acontece com livros considerados clássicos ou eruditos.

Sendo assim, a ficção científica cresce como um gênero literário de massa. Além de popular, ele foi capaz de transitar entre múltiplos espaços e mídias, mantendo seu caráter volátil, mutante e híbrido. A ficção científica se torna, portanto, um meio muito adequado para a veiculação das inúmeras representações, significações e ambiguidades, constantemente ultrapassando as fronteiras limitadoras das formas, o tempo, o espaço.

\section{THE GENRE (?) OF SCIENCE FICTION}

ABSTRACT: This article aims at the problematization of science fiction as a literary genre. A historical contextualization of the works of science fiction is followed by the presentation, exemplification and analysis of elements which James e Mendlesohn (2003) and Gary Westfahl (1999) consider as "immediately recognized" in sci-fi works, therefore debating the considerations and disconsiderations of science fiction as a literary genre. This article also deals with the fact science fiction is questioned as a genre because it is part of a mass popular culture, not erudite, focusing on the great outreach of sci-fi, valuing its capacity of transiting among multiple spaces and media, keeping its volatile, mutant and hybrid character.

KEYWORDS: Science fiction genre. Mass culture. Pulp magazines.

\section{REFERÊNCIAS}

ALDISS, Brian Wilson. Billion years spree: the true story of science fiction. New York: Schocken Books, 1976. 
BRANTLINGER, Patrick. The gothic origins of science fiction. NOVEL: a forum on fiction, [S.I]: Brown Unibersity Press, v. 14, n. 1, outono 1980, p. 30-43.

CHILDERS, Joseph e HENTZI, Gary (Org.). Columbia dictionary of modern literary and cultural criticism. Nova York: Columbia University Press, 1995.

CLOUGH, B. W. Provisional Solutions. In: SAKERS, Don (Org.). Carmen Miranda's ghost is haunting space station three. Nova York: Baen Books, 1990.

CSICSERY-RONAY JR., Istvan. On the grotesque in science fiction. Science fiction studies, [S.I]: SF-TH Inc, v. 29, n. 1, p. 71-99 mar. 2002.

DE LA ROCQUE, Lucia; TEIXEIRA, Luiz Antonio. Frankenstein, de Mary Shelley e Drácula, de Bram Stoker: gênero e ciência na literatura. História, ciências, saúde, Manguinhos: [s.n.], v. VIII(1), p. 10-34, mar.-jun. 2001.

PHILMUS, Robert M. Into the unknown: the evolution of science fiction from Francis Godwin to H. G. Wells.Berkeley: University of California Press, 1970.

GREWEL, Greg. Colonizing the universe: science fictions then, now, and in the (imagined future). Rocky Mountain review of language and literature [S.I.: s.n], v. 55, n. 2, p. 25-47, 2001.

HOLLINGER, Veronica. (Re)reading queerly: science fiction, deminism, and the Defamiliarization of gender. Science fiction studies, [S.I]: SF-TH Inc, v. 26, n. 1, pp. 23-40, mar.1999.

Feminist theory and science fiction. In: MENDLESOHN, Farah; JAMES, Edward (Org.). The Cambridge companion to science fiction. Cambridge: Cambridge University Press, 2003.

LEM, Stanislaw, et al. On the structural snalysis of science fiction. Science fiction studies, [S.I.]: SF-TH Inc, v. 1, n. 1, p. 26 33, primavera 1973.

Looking down on science fiction: a novelist's choice for the world's worst writing. Science fiction studies, [S.I]: SF-TH Inc, v. 4, n. 2, p. 127-128, jul.1977.

LEVINE, Lawrence W. Highbrow/Lowbrow: the emergence of cultural hierarchy in America. Boston: Harvard University Press, 1998.

LUCKHURST, Roger. Border policing: postmodernism and science fiction (Surveillant les frontiéres: le postmodernisme et la SF). Science Fiction Studies, [S.I] SF-TH Inc v. 18, n. 3, p. 358-366, nov. 1991.

JAMES, Edward e MENDLESOHN, Farah; (Org.). The Cambridge companion to science fiction. Cambridge: Cambridge University Press, 2003.

RUSS, Joanna. Towards an aesthetic of science fiction. Science fiction studies, [S.I]: SF-TH Inc, v. 2, n. 2, p. 112-119, jul. 1975. 
SAKERS, Don. Carmen Miranda's ghost is haunting space station three. Nova York: Baen Books, 1990.

SOARES, Angélica. Gêneros literários. São Paulo: Ática, 1997.

SFE. Pulp. 3 de abril 2015. SFE: The Encyclopedia of Science Fiction. Disponível em: <http://www.sf-encyclopedia.com/entry/pulp>. Acesso em: 5 de abril 2018.

SHWARTZ, S. Confessional Booths. In: SAKERS, Don (Org.). Carmen Miranda's ghost is haunting space station three. Nova York: Baen Books, 1990.

SUVIN, Darko. On the poetics of science fiction genre. College English.

Bloomington: Indiana University, v. 34, n. 3, dez. 1997. p. 372-382.

WESTFAHL, Gary. The popular tradition of science fiction criticism, 1926-1980. Science fiction studies, [S.I]: SF-TH Inc, v. 26, n.2, p. 187-212, jul. 1999. 